Research Article

\title{
IMPEDANCE IMAGE RECONSTRUCTION WITH ARTIFICIAL NEURAL NETWORK IN ELECTRICAL IMPEDANCE TOMOGRAPHY
}

\section{Beyhan KILIC ${ }^{*}$}

Electrical impedance tomography views the electrical properties of the objects by injecting current with surface electrodes and measuring voltages. Then using a reconstructing algorithm, from the measured voltage-current values, conductivity distribution of the object calculated. Finding internal conductivity from surface voltage-current measurements is a reverse and illposed problem.

Therefore, high error sensitivity, and making approximations in conceiving complex computations cause to limited spatial resolution. The classic iterative image reconstruction algorithms have reconstruction errors. Accordingly, Electrical impedance tomography images suffer low accuracy. It is necessary to evaluate the collected data from the object surface with a new approach. In this paper, the forward problem solved with the finite element method to reconstruct the conductivity distribution inside the object, the reverse problem solved by the neural network approach. Image reconstruction speed, conceptual simplicity, and ease of implementation maintained by this approach.

Key words: electrical impedance tomography, finite element methods, biomedical image reconstruction, neural network

\section{Introduction}

Electrical impedance tomography (EIT) creates the object's internal electrical conductivity or resistivity by injecting current with electrodes on the surface, and the corresponding voltages are measured with electrodes using the neighboring method. The measured current and voltages values are used to reconstruct the object's internal conductivity distributions [1].

The image of conductivity distribution is constructed with the iterative process by measured current and voltages. The first step is to find voltage and current distributions to create an impedance profile. This procedure is defined as " the forward problem." to reconstruct the electrical conductivity distributions inside the object from the knowledge of the measured electrode voltages and applied current is an inverse problem [2]. Imaging the internal impedance distribution by processing voltagecurrent data, which is measured form objects surface, is a nonlinear and ill-posed inverse problem. The none-linearity of the problem requires a regularization, but the linearization approximation is incapable of reconstructing the objects that have complex geometries or to a large extent, contrasts. Therefore an Iterative solution is inevitable.

\footnotetext{
${ }^{1}$ Faculty of Electrical and Electronics, Department of Electrical Engineering, Yildiz Technical University, Istanbul, Turkey, (beykilic@yildiz.edu.tr) (iD https://orcid.org/0000-0002-8438-8369 
The most common non-linear method is Newton-Raphson. This technique works by searching the conductivity distribution that reduces the difference between applied potentials and the calculated potential from the forward problem by the finite element method. The drawback of this algorithm is that it is computationally complex, requires numerous orders of magnitude and computational time is high as compared to the linear methods [3].

EIT is entirely non-invasive and radiation-free. EIT has an advantage over other imaging techniques, such as its portability, low-cost, and faster imaging capabilities [4]. EIT is suitable for medical imaging of the body and pathological situations [5-6]. Though advantages, EIT technology suffers lower spatial resolution in comparison to other imaging systems like magnetic resonance imaging (MRI) and computed tomography (CT). For Improving spatial resolution, electrode numbers can be increased, but implementing a lot of electrodes can not be possible in most real situations [7].

\section{EIT Fundamentals}

Impedance or resistivity is defined as the current and voltage ratio [8]. The human cell is resistive, piecewise homogeneous, and linear volume conductor. Different tissue has different resistivity value. Tissue resistivity values are shown in Tab. 1. The blood resistivity depends strongly on hematocrit (Hct) [9]. The EIT converts the different electrical properties of biological tissues to critical information for pathological diagnosis situations.

Table1. different biological tissue resistivity values

\begin{tabular}{ll}
\hline Tissue & Resistivity Values \\
\hline Blood & $1.6 \Omega \mathrm{m}$ \\
Heart & $2.5 \Omega \mathrm{m}$ \\
Lungs & $20 \Omega \mathrm{m}$ \\
Bone & $177 \Omega \mathrm{m}$ \\
Fat & $25 \Omega \mathrm{m}$ \\
\hline
\end{tabular}

The first step is to determine voltage-current distributions from the object surface for imaging impedance. This problem is defined as "the forward problem" for a given impedance profile voltagecurrent values. The results of the forward problem and the measured values used to impedance values. Finally, impedance images are reconstructed. The second step is to reconstruct impedance images and named inverse problem. Impedance image reconstruction process is shown in Fig. 1.

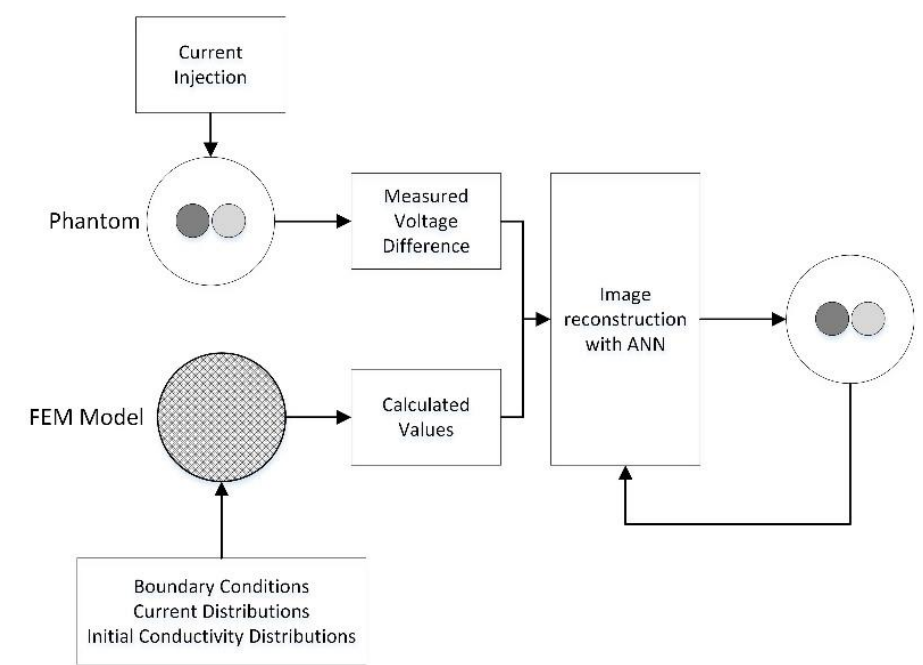

Figure 1. Block diagram for the image reconstruction process in EIT 
Electrodes used in medical imaging are generally found as the surface electrodes in most of the cases. In other fields, the electrodes can be soldered or penetrated to the object. The injected current amplitude and frequency are required to be chosen very carefully for the medical EIT, whereas the magnitude could be increased more for the non-biological sample imaging with EIT. Contact impedance is found a crucial issue in EIT in most of the applications, and hence the four-electrode method is preferred.

\section{The Solution of The Forward Problem}

Measuring the voltages from the object"s or phantom surface is defined as the forward problem. Solving the forward problem is to collect data from the subject's surface or phantom. The current or voltage is applied to the object, and the resulting current or voltage is calculated. Typically, the current is applied from an alternating current (AC) source to a few electrodes, and the measuring voltages are calculated to form the remaining electrodes. Voltage measured from the other electrode pair by the principle of the neighboring method [9]. The current is injected into pairs of electrodes numbers one and second. Subsequently, the voltage is measured between the electrode pairs three and four and so on $[10]$.

\subsection{Additional instructions (Word Style TS Heading 2)}

The forward problem can be solved analytically for simple objects but in the case of the complex objects. The domain is discretized into small elements called mesh or finite elements. The problem is defined for a continuous domain; therefore, area equations are valid for all finite elements. The resistivity of each finite element is assumed homogenous. While the size of the finite element goes to zero, the solution approaches to the real solution. The mathematical expressions of the FEM are welldefined in literature [11-12-13]. The continuous domain is found by attaching the finite elements. Each element has two node numbers: local and global. The finite elements are unified by common nodes.

$K_{\mathrm{n}}$ element matrix and $Q_{\mathrm{n}}$ vector are unified as a master matrix. $K_{\mathrm{n}}$ element matrix is given in Eq. 1. The problem is converted to a linear problem form differential equation by modeling discrete finite element a continuous domain.

$$
\begin{array}{ll}
K \emptyset=\mathrm{Q} & \\
\text { Where; } & \\
K_{\mathrm{nxn}} & : \text { master matrice } \\
\mathrm{n} & : \text { node number in mesh } \\
\varnothing & : \text { node voltage vector } \\
\mathrm{Q} & : \text { node current vector }
\end{array}
$$




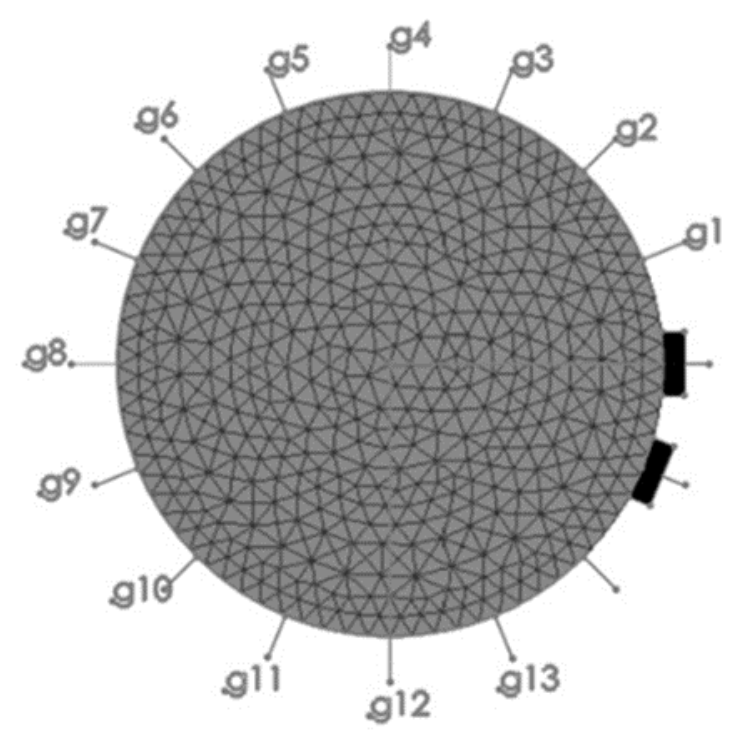

Figure 2. Finite element model

In this study, the domain is separated into 1016 finite triangle elements and 541 nodes. Figure 2 shows the domain. The master matrice is solved with the frontal solution. The method was developed by Irons B.M [14], and Ider at al. modified it [15-16]. The master matrix is solved during the matrix generation stage before the matrix is completed. The frontal length of the matrix depends on mesh structure and the numbering technique of nodes. In a well-defined mesh, the frontal size of the mesh shouldn't change extremely. The test mesh consisting of 541 nodes and 1016 finite elements is shown in Fig. 2. The potential variations of the studied domain are known approximately. The system contains sixteen electrodes. Two of them are current injection electrodes, remaining are voltage measurement electrodes, and the conductivity is assumed to be $0.002 \mathrm{~S} / \mathrm{cm}$. For sixteen different boundary conditions, for $\mathrm{I}=5 \mathrm{~mA}$, the problem is solved synchronically. The measured voltage values which are used to test the accuracy of the calculated solution are given in Tab. 2 .

Tablo 2. The calculated and the measured voltage differences of the test set

\begin{tabular}{ccc}
\hline Difference voltage & Measured value $(\mathbf{m V})$ & Calculated value $(\mathbf{m V})$ \\
\hline V1 & 47.8695 & 47.901628 \\
V2 & 20.84387 & 20.906145 \\
V3 & 12.53245 & 12.588936 \\
V4 & 8.947047 & 8.99501696 \\
V5 & 7.202337 & 7.244238 \\
V6 & 6.382648 & 6.421197 \\
V7 & 6.155331 & 6.192814 \\
V8 & 7.229110 & 6.448648 \\
V9 & 8.967458 & 7.270297 \\
V10 & 12.534843 & 9.013852 \\
V11 & 12.605730 & 12.588936 \\
V12 & 20.906145 & 20.923509 \\
V13 & 47.734701 & 47.704444 \\
\hline
\end{tabular}




\subsection{The Inverse Problem}

The reconstruction of the image of the objects inside by using data measured from the object surface is an ill-posed problem. As a result of this ill-condition, the image reconstruction system is highly sensitive to measurement errors, and spatial resolution is low, especially in the center of the object. Besides, to simplify the calculation complexity, some assumptions must be made.

Each assumption is accurate for special conditions. These assumptions limit the accuracy of the solution. Therefore, the image reconstruction problem comes across poor accuracy and limited spatial resolution. In the electrical impedance image reconstruction process, it is necessary for a new approach to evaluate the data measured from the surface of the object. The Artifical Neural Network ANN method is suitable for this process because ANN consists of neurons with weighted value. There is a structural correlation between ANN and FEM. The neurons are matched with finite elements. The conductivity distribution is modeled by finite elements as given in Eq. 2.

$$
C(n)=\sum_{i=1}^{1} b(n, i) * u(i)
$$

Where;

$C(n), n=1,2, \ldots, N$, different conductivity numbers

$u(i), \quad i=1,2, \ldots ., 256$, for different projections, 16 voltage differences measured from the surface.

$b(n, i)$, element weightiness matrice.

The weight matrix shows the best approach in terms of least squares from voltage measurements to conductivity changes. The weight matrix is calculated iteratively during the training of the ANN. The error backpropagation algorithm is used this prosses.

In this study, the ANN used to provide the recognition of conductivity changes from $16 \times 16=256$ voltage differences taken from the circular disc surface with a radius of $15 \mathrm{~cm}$ is shown in Fig. 1.

The voltages differences taken from the surface applied to the ANN input, the conductivity patterns are recognized by the multi-layer ANN. During the network training process, inputs and desired outputs are used for training. The learning process is realized through changing of the elements of the weight. Here, the backpropagation algorithm of the error is used. The difference between the instantaneous output values and the target output, the error is backpropagated across the network, changing the link weights. This calculation is iterated until the desired error value is reached.

\subsection{Simulation of Conductivity Patterns}

To solve the inverse problem, an ANN used to form the analogy from voltages difference to conductivity changes. For this purpose, the 1016-finite elements network used in the forward solution is divided into 16-element groups and turned into a coarse network [17]. The voltage difference calculated by the finite element method in the forward solution is normalized in the range $(0-1)$ and applied to the mesh input layer. The network was trained, 64 different conductivity values were matched with the model given in Fig. 2. Since there is no need to make a mathematical definition between the input layer and the output layer in ANN, the problem is both conceptually simplified, and the solution is reached faster. A trained network can recognize different conductivity patterns in seconds. The ANN was trained on the circular finite element geometry with 64 finite elements. The Ankara Standard Data, 16 sets of voltages, were used to test the performance of the net [10]. The test object is a 2D circular disk. The number of the input layer is selected as104 and the output layer 64 . The number of a hidden 
layer of the network is two. In the stage forward problem solution. At the end of 81340 iterations, $0.259 \%$ error rate was achieved.

In Figure 3a and Fig. 3b, the conductivity distribution graph obtained with ANN for the case where the object with conductance value of $0.002 \mathrm{~S} / \mathrm{cm}$ is in the center of the phantom is shown. The reconstructed conductivity images for two objects with a conductivity of $0.02 \mathrm{~S} / \mathrm{cm}$ between the center and the surface are given in Fig. 4a and in Fig. 4b. The conductivity image obtained by ANN with two objects having a conductivity of $0.02 \mathrm{~S} / \mathrm{cm}$ was obtained with $0.05 \%$ error. The conductivity distribution of the object having a conductance of $0.02 \mathrm{~S} / \mathrm{cm}$ between the phantom center and the surface is given in two dimensions in Fig. 5a and in three dimensions in Fig. 5b. The conductivity image was obtained with $0.03 \%$ error.

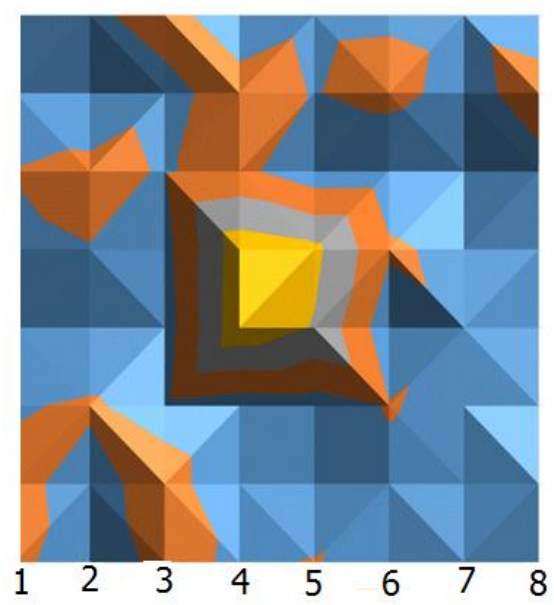

a)

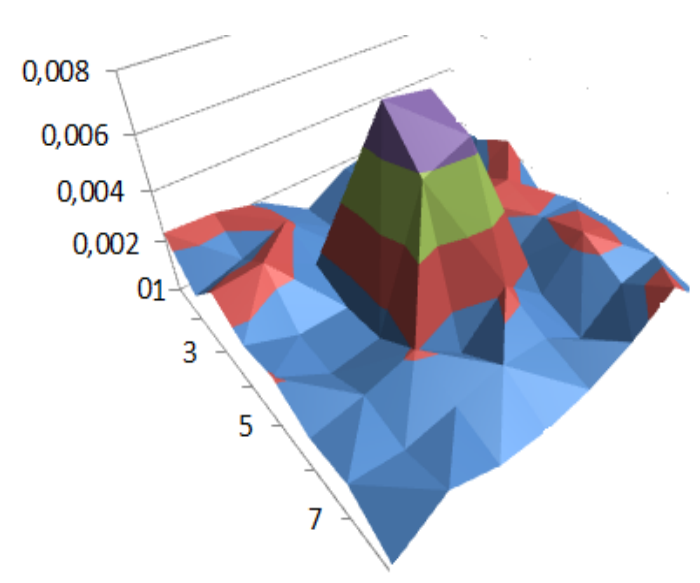

Figure 3. a) Two dimensional reconstructed conductivity image, error $\% 0,02$ b) Two dimensional reconstructed conductivity image, error \% 0,02
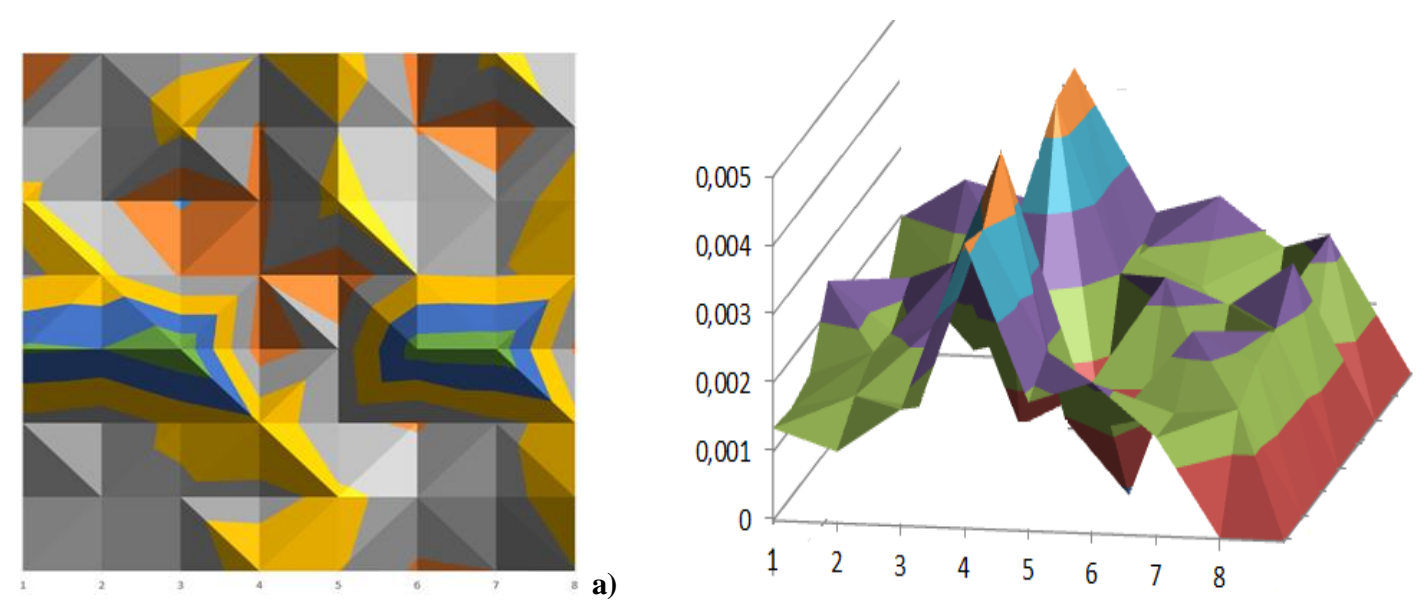

b)

Figure 4. a) Two-dimensional reconstructed conductivity image of two objects, error $\% 0,05$ b) Three dimensional reconstructed conductivity image of two objects, error $\% 0,05$ 

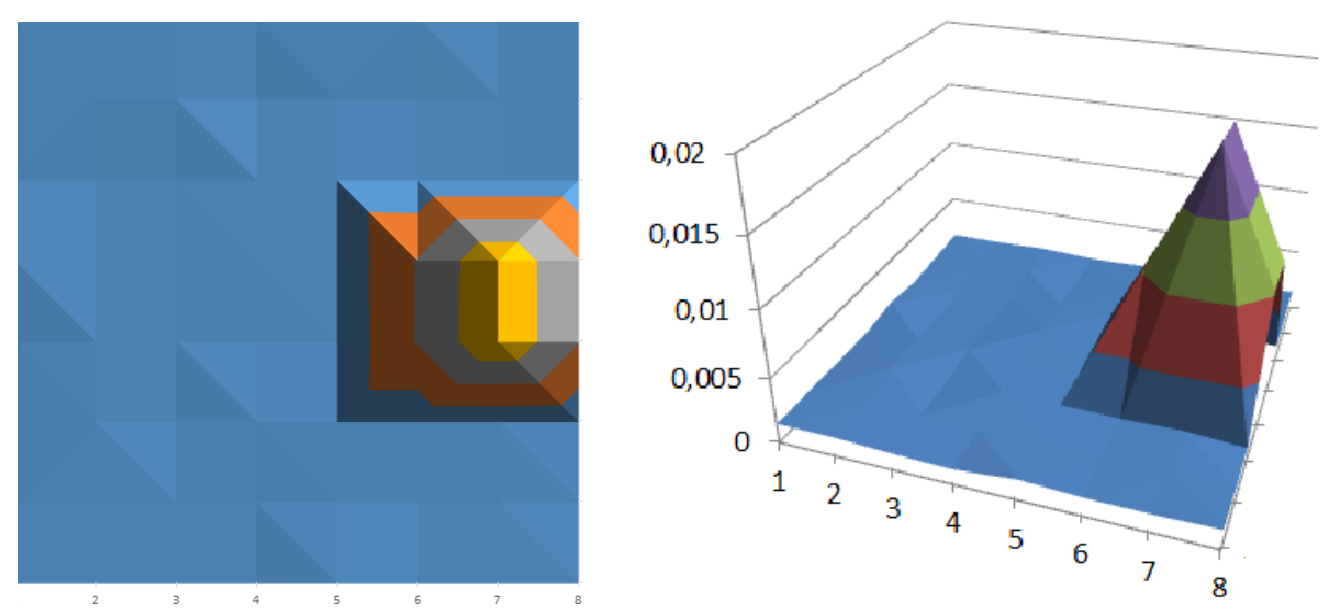

Figure 5. a) Two dimensional reconstructed conductivity image, error $\% 0,03$ b) Two dimensional reconstructed conductivity image, error $\% 0,3$

\section{Conclusion}

EIT is a non-invasive, and with no potential hazard imagining tool for monitoring medical application. It is widely used in numerous medical imaging. In this paper, steps to solve the EIT problem are briefly discussed. FEM in the forward solution and the artificial neural network (ANN) in inverse solution.

In this paper, ANN has been proposed to generate images of conductivity changes from the phantom surface area measurements on electrical impedance imaging. The forward problem was solved with FEM so that the problem was solved directly using ANN. Since FEM and ANN have structural matching, in reverse problem solving, there is no need to make new assumptions, therefore error rate decreases. The conductivity contrast was found to be approximately equal to one. The resolution will be reduced in a multi-conductivity environment where there are significant variations in the conductivity. EIT can be preferred for monitoring patients. After the completion of training in artificial neural networks, it is possible to perform automatic data analysis with ANN, since results are obtained in a short time.

\section{References}

[1] Adler, A., Guardo, R., (1994). A Neural Network Image Reconstruction Technique for Electrical Impedance Tomography. IEEE Transactions on Medical Imaging, 13(4).

[2] Martin, S., Choi, C.T.M., Electrical Impedance Tomography: A Reconstruction Method Based on Neural Networks and Particle Swarm Optimization, Springer, Cham, Switzerland, 2015.

[3] Khan, T.A., Ling, S.H., (2019). Review On Electrical Impedance Tomography. Artificial Intelligence Methods and its Applications Algorithms,12(5), 88, 1-18.

[4] Webster, J. G., Electrical Impedance Tomography, Adam Hilger Series of Biomedical Engineering, Adam Hilger, New York, USA, 1990.

[5] Hikmah, A. (2019). Two-Dimensional Electrical Impedance Tomography (EIT) For Characterization of Body Tissue Using a Gauss-Newton Algorithm, OP Conf. Series: Journal of Physics: Conf. Series, 1248 
[6] Miller, A., Blott, S., et al. (1992). Review of Neural Network Applications in Medical Imaging and Signal Processing. Medical and Biological Engineering and Comp., 30(5), 449-464

[7] Malmivuo, J., Plonsey, R., Bioelectromagnetism Principles and Applications of Bioelectric and Biomagnetic Fields, Oxford Scholarship, 1995.

[8] Uhunmwangho, R., Ibo, A.O., Introduction To Electrical Engineering, Odus Press, 2017

[9] Graham, B.M., Enhancements in Electrical Impedance Tomography (EIT) Image Reconstruction for Three-Dimensional Lung Imaging, Ph.D. thesis, University of Ottawa, 2007

[10] Ankara Standard Data Set, European Community Concerned Action in Impedance Imaging, Image Reconstruction Meeting, Oxford, UK, 14-17, 1994

[11] Pursiainen, S., Hakula, H., (2006). A High-order Finite Element Method for Electrical Impedance Tomography, Progress In Electromagnetics Research Symposium, Cambridge, 57-62.

[12] Garnadi, A.D., (1997). Electrical Impedance Tomography Based on Mixed Finite Element Model, Proceedings CMSE'97, Bandung, 4, 6-7.

[13] Woo, E.J., Hua, P., et al. (1994). Finite-Element Method in Electrical Impedance Tomography, Medical and Biological Engineering and Computing, 32(5), 530-536.

[14] Irons, B.M., (1970) A Frontal Solution Program for Finite Element Analysis, International Journal for Numerical Methods in Engineering, 2, 25-32.

[15] Ider,Y.Z., Gencer, N., et al. (1990). Electrical Impedance Tomography of translationally uniform cylindrical objects with general cross-sectional boundaries, IEEE Medical Imaging, 9(1), 49-59.

[16] Ider, Y.Z., Nakiboğlu, B., et al. (1992). Determination of The Boundary of an Object Inserted Into a Water-filled Cylinder, Clinical Physics and Physiological Measurement 13(A), 151-154

[17] K1lıç, B., Elektrik Empedans Tomografisinde Sonlu Eleman Yöntemi ile Modelleme ve Görüntü Oluşturma Agoritmaları, Doktora Tezi (Ph.D. thesis), Yıldız Teknik Üniversitesi, 1998. 